\title{
Appraisal of Principals' Role Performance and the Teachers' Perception of the Same Roles in Selected Public Schools in Osun State, Nigeria
}

\author{
Dr. F. B. Bamire ", Prof. M. A. Adelabu \\ Department of Educational Management, Obafemi Awolowo University, Ile-Ife, Nigeria
}

*Corresponding Author: Dr. F. B. Bamire, Department of Educational Management, Obafemi Awolowo University, Ile-Ife, Nigeria

\begin{abstract}
The paper appraises the principals' role and the perception of teachers in selected public secondary schools in Osun State of Nigeria. The paper specifically investigates the principals' role performance and the teachers' perception of the same role (instructional, administrative and human relations) in the selected public secondary schools. The survey research design was used for the study. The population consisted of the principals and the teachers in public secondary schools in Osun State, Nigeria. Altogether 210 respondents were sampled comprising 175 teachers and 35 principals randomly selected from the six geo-political zones (Iwo, Ikirun, Ife, Ilesa, Osogbo and Ede) in Osun State. Thirty-five (35) schools were randomly selected from the list of all the secondary schools in the six zones in Osun State. Two sets of questionnaire were used for data collection. These were: Principals' Role Performance Questionnaire and Teachers' Rating of the Principals' Role Performance Questionnaire. The data collected were analysed using descriptive statistics. The results showed that about $78 \%$ of the principals claimed to perform the administrative role very well while $62.5 \%$ and $59.4 \%$ performed the human relations and the instructional roles respectively. However, the rating of teachers showed that only $37.5 \%$ of the principals actually performed the instructional role, $70.9 \%$ performed the administrative role while $64.5 \%$ performed the human relations role in the schools. The general view of the teachers on the principals' actual performance of their role is that principals perform more of administrative role and human relations role than instructional role in the school. It was concluded that promoting and intensifying the instructional role of the principal is capable of enhancing the students' academic performance in schools.
\end{abstract}

Keywords: Principals, Role Performance, Teachers Perception, and Public Schools

\section{INTRODUCTION}

According to recent research, Secondary education in Nigeria has been enduring deterioration in the quality of secondary school education delivery in Nigeria and as a result, school success is not achieved (Nigeria Education Sector Analysis (NESA), 2014). There is a general belief that the standard of education in Nigeria has dropped and continues to drop. This is evident in the inability of some secondary school leavers in Nigeria to do what a primary school leaver could do effectively some decades ago, such as writing good essays (Usman, 2009). At the university level, the story is not different as many graduates are incompetent and not resourceful. Employers do not have confidence in them any longer, as they are nostalgic about graduates of years ago because of the then high quality of education.

As a result of the falling standard and quality of education at secondary level of education in Nigeria, principals were indicted by the parents, the ministry of education and the society at large. For instance, River state Government went as far as querying the principals in his state over the poor performance in public examinations (Oyebade, 2014). Over the years principals have been criticized for non-performance of their roles. They have been said to be inefficient and ineffective in their role performance as they fail to provide direction for their teachers.

The poor performance in Public examination of Nigeria secondary school students was also evident in the level of failure (61.32\%) in the 2015 May/June Senior Secondary Certificate Examination (SSCE) conducted by West African Examination Council (WAEC, 2015). Schools do not seem to foster educational, social, emotional and moral development anymore, as there were cases of drop-out, 
examination malpractice, truancy and immoral behaviours witnessed among the secondary school students. The familiar explosion of knowledge and student population has resulted in an increase in teachers' population and the role of the principal has come to assume wider and more complex dimension. In an attempt to redress these problems, a lot of responsibility is placed on the school principal, which he is expected to perform to the best of his capability, hence the need to examine the extent to which the principal performs such roles in secondary schools.

Many notable scholars such as Niewman and Hughes (1951), Gross et al (1966), Biddle and Thomas (1966) and Adelabu (1981) have tried to promote an acceptable definition of role. Although there are variations in usage, there is a commonality in the meaning of role. This study adapts the definition of role according to Deighton (1971) that role concerns itself with the behaviour of people and its relation to the behavior of others (i.e. his role sets) in a social system. This paper considers the principal as the role incumbent while his role set is the teacher. This role set is in a good position to appraise the principal's day-to-day performance of his job. The principal may not be able to see a marked difference between the teacher's expectation and the actual performance of his roles; but, his role set (the teachers) is in the best position to appraise his performance such that if there is a difference between the teachers' expectation and the principal's actual performance of his role then there is role conflict.

Over the last decade, studies of organizations in general, and educational organizations in particular, have consistently found role conflict to be an important factor to contend with in a work place (Rizzo et al, 1970). For workers in such organizations, there is substantial evidence to suggest that role conflict may have negative consequences with regard to employee's wellbeing, job and career satisfaction, turn over intentions and overall organizational effectiveness (Bacharach and Bamberger, 1996). One of the most common sources of conflict for the principal is the divergence among the expectations of different people such as the teachers, students, parents and others to whom the principal enacts his role (Uwazurike, 1992; Adeyegbe, 1992). All these people who constitute the role sets of the principal may have their own ideas as to what the principal should do and not do. Their ideas often differ from the principal's own definition of his role, and the situation may become complicated if the principal interprets his actual expectations differently.

There is a wide gap between what was and what is currently existing in the public schools and in order to normalize this situation, the school principal should be at his best to enhance the academic performance of his students. There is however a dearth of information on the appraisal of principal's role performance in Osun State, Nigeria.

\subsection{The Research Problem}

Nigerians have come out open to criticize the falling standard of education in the country and Osun State citizens in particular have also expressed great concern over the poor performance of students in public examinations over the years. Evidence from Adaralegbe (1983); Ajeyalemi and Ejiogu (1987), shows that the role performance of the school principals has a major impact on the overall effectiveness and good performance of the school. It is important to note that an average Nigerian principal does not seem to be conscious of the various roles expected of him; and if he is aware, the extent to which these roles are performed becomes of tremendous concern. Research evidence (Afolabi, 1998) on Nigerian secondary schools as well as personal interactions with some schools in Osun state, in particular, revealed that the expectations of the teachers were at variance with the role performance of the principals most of the time. This divergence has adversely affected the attainment of the educational objectives of the school.

For instance, if the principal is expected by his teacher to take an action, and the principal does not perceive that such an action should be taken, the teacher concerned may not be happy and this may affect his work in school. The principal may view his role differently from how the teacher views it as teacher may have his own expectations regarding the principal's role performance. This is a fundamental problem which needs to be given urgent attention hence this study.

\subsection{Objectives of the Study}

This paper investigates principals' role performance and the teachers' perception of the same roles in selected public secondary schools in Osun State, Nigeria. Specifically, the objectives of the study were to: 
- Investigate teachers' perception of the principals' actual performance of their instructional roles

- Investigate teachers' perception of the principals' actual performance of their administrative roles

- Investigate teachers' perception of the principals' actual performance of their human relation roles

- Determine teachers' rating of the principals' actual performance of their instructional roles

- Determine teachers' rating of the principals' actual performance of their administrative roles

- Determine teachers' rating of the principals' actual performance of their human relation roles.

\subsection{Research Questions}

- How do teachers perceive principals' actual performance of their instructional roles?

- How do teachers perceive principals' actual performance of their administrative roles?

- How do teachers perceive principals' actual performance of their human relation roles?

- What is teachers' rating on the principals' actual performance of their instructional roles?

- What is teachers' rating on the principals' actual performance of their administrative roles?

- What is teachers' rating on the principals' actual performance of their human relation roles?

\section{Methodology}

The survey research design was used for the study. The survey was conducted in the six geopolitical zones (Iwo, Ikirun, Ife, Ilesa, Osogbo and Ede) in Osun State, Nigeria. A list of all the public secondary schools in the state was obtained from the state's Ministry of Education, from where 35 schools were randomly selected in proportion to the number of schools in each of the six geopolitical zones. Five teachers, who had worked with the principal for at least two academic sessions were purposively selected in each of the schools to give a total of 175 teachers while all the 35 principals in the selected schools were involved. Altogether, 210 respondents were selected for the study based on the zones with the highest number of schools, Focus Group Discussion (FGD) was also held in four of the six geopolitical zones of the state. A group of male and female teachers who have experience about the school were interviewed separately in order to gain better insight and understanding into the principals' role performance.

Two sets of structured questionnaire were constructed for the study; the first set of questionnaire was on principals' role perception in secondary schools while the second set was on actual role performance of principals in the schools. The two sets of questionnaires were designed for the principals and the teachers who have been selected for the study. While the first set of questionnaire focused on role expectation of secondary school principals, the second was on the actual role performance of principals. A five-point Likert-type rating scale was adopted for quantifying responses to the items in the first set of questionnaire relating to the principals' expected roles, consisting of strongly Agree (SA), Agree (A), Undecided (U), Disagree (D) and Strongly Disagree (SD). To quantify responses to the items in the second set of questionnaire relating to principals' actual performance of their roles, a modified three-point Likert-type rating scale was used.

Data generated were analysed using the statistical package for social science (SPSS) and based on the research questions raised, descriptive statistics was employed to organize the data and mean responses were used to determine the role performance of the principals in different functional areas considered in the study. These functional areas are: instructional role, administrative role and human relation role of the school principal.

\section{RESULTS AND DISCUSSION}

The results of analysis on how teachers perceive principals' performance of their instructional, administrative and human relation roles are presented in Table 1. 
Appraisal of Principals' Role Performance and the Teachers' Perception of the Same Roles in Selected Public Schools in Osun State, Nigeria

Table1. Teachers perception of the principals' actual performance of their instructional roles

\begin{tabular}{|c|c|c|c|c|c|c|c|}
\hline \multirow[t]{3}{*}{$\mathbf{Q} / \mathbf{N}$} & \multirow[t]{3}{*}{ Instructional Role } & \multicolumn{3}{|c|}{ Teachers Perception } & \multicolumn{3}{|c|}{ Principals Actual Performance } \\
\hline & & Positive & Neutral & Negative & Satisfactory & Fair & Poor \\
\hline & & $\begin{array}{l}\text { Freq. } \\
(\%)\end{array}$ & $\begin{array}{l}\text { Freq. } \\
(\%)\end{array}$ & $\begin{array}{l}\text { Freq. } \\
(\%)\end{array}$ & $\begin{array}{l}\text { Freq. } \\
(\%)\end{array}$ & $\begin{array}{l}\text { Freq. } \\
(\%)\end{array}$ & $\begin{array}{c}\text { Freq. } \\
(\%)\end{array}$ \\
\hline 1 & Give demonstration lessons & $\begin{array}{l}133 \\
(67.8)\end{array}$ & $\begin{array}{l}13 \\
(7.6)\end{array}$ & $\begin{array}{l}25 \\
(14.6)\end{array}$ & $\begin{array}{l}20 \\
(62.5)\end{array}$ & $\begin{array}{l}9 \\
(28.1)\end{array}$ & $\begin{array}{l}3 \\
(9.4)\end{array}$ \\
\hline 2 & $\begin{array}{l}\text { Provide badly needed } \\
\text { instructional materials }\end{array}$ & $\begin{array}{l}155 \\
(90.7)\end{array}$ & $\begin{array}{l}4 \\
(2.3)\end{array}$ & $\begin{array}{l}12 \\
(7.0)\end{array}$ & $\begin{array}{l}17 \\
(53.1)\end{array}$ & $\begin{array}{l}15 \\
(46.9)\end{array}$ & - \\
\hline 4 & $\begin{array}{l}\text { Ensure that teachers improve on } \\
\text { the use of instructional materials }\end{array}$ & $\begin{array}{l}156 \\
(91.3)\end{array}$ & $\begin{array}{l}8 \\
(4.7)\end{array}$ & $\begin{array}{l}7 \\
(4.1)\end{array}$ & $\begin{array}{l}19 \\
(59.4)\end{array}$ & $\begin{array}{l}13 \\
(40.6)\end{array}$ & - \\
\hline 7 & $\begin{array}{l}\text { Organize orientation course } \\
\text { for new teachers }\end{array}$ & $\begin{array}{l}115 \\
(67.3)\end{array}$ & $\begin{array}{l}29 \\
(17.0)\end{array}$ & $\begin{array}{l}27 \\
(15.8)\end{array}$ & $\begin{array}{l}11 \\
(34.4)\end{array}$ & $\begin{array}{l}13 \\
(40.6)\end{array}$ & $\begin{array}{l}8 \\
(25.0)\end{array}$ \\
\hline 10 & $\begin{array}{l}\text { Encourage teachers to attend } \\
\text { seminars }\end{array}$ & $\begin{array}{l}155 \\
(90.6)\end{array}$ & $\begin{array}{l}6 \\
(3.5) \\
\end{array}$ & $\begin{array}{l}10 \\
(5.9) \\
\end{array}$ & $\begin{array}{l}14 \\
(43.8)\end{array}$ & $\begin{array}{l}18 \\
(56.3)\end{array}$ & - \\
\hline 13 & $\begin{array}{l}\text { Undertake inter-schools } \\
\text { visitation }\end{array}$ & $\begin{array}{l}126 \\
(73.7)\end{array}$ & $\begin{array}{l}23 \\
(13.5)\end{array}$ & $\begin{array}{l}22 \\
(12.9)\end{array}$ & $\begin{array}{l}8 \\
(25.0)\end{array}$ & $\begin{array}{l}20 \\
(62.5)\end{array}$ & $\begin{array}{l}4 \\
(12.5) \\
\end{array}$ \\
\hline 16 & $\begin{array}{l}\text { Help the inexperienced } \\
\text { teachers }\end{array}$ & $\begin{array}{l}120 \\
(70.2)\end{array}$ & $\begin{array}{l}29 \\
(17.0)\end{array}$ & $\begin{array}{l}22 \\
(12.9)\end{array}$ & $\begin{array}{l}15 \\
(46.9)\end{array}$ & $\begin{array}{l}14 \\
(43.8)\end{array}$ & $\begin{array}{l}3 \\
(9.4)\end{array}$ \\
\hline 19 & $\begin{array}{l}\text { Occasionally collect random } \\
\text { samples of students' exercise } \\
\text { books }\end{array}$ & $\begin{array}{l}144 \\
(84.3)\end{array}$ & $\begin{array}{l}16 \\
(9.4)\end{array}$ & $\begin{array}{l}11 \\
(6.4)\end{array}$ & $\begin{array}{l}15 \\
(46.9)\end{array}$ & $\begin{array}{l}16 \\
(50.0)\end{array}$ & $\begin{array}{l}1 \\
(3.1)\end{array}$ \\
\hline 25 & $\begin{array}{lrr}\text { Summon } & \text { meetings } & \text { of } \\
\text { departmental } & \text { heads } & \text { to } \\
\text { coordinate instructions } & \end{array}$ & $\begin{array}{l}159 \\
(93.0)\end{array}$ & $\begin{array}{l}8 \\
(4.7)\end{array}$ & $\begin{array}{l}4 \\
(2.4)\end{array}$ & $\begin{array}{l}23 \\
(71.9)\end{array}$ & $\begin{array}{l}8 \\
(25.0)\end{array}$ & $\begin{array}{l}1 \\
(3.1)\end{array}$ \\
\hline 28 & $\begin{array}{l}\text { Organize in-service training } \\
\text { for teachers }\end{array}$ & $\begin{array}{l}108 \\
(63.2)\end{array}$ & $\begin{array}{l}31 \\
(18.1)\end{array}$ & $\begin{array}{l}32 \\
(18.7)\end{array}$ & $\begin{array}{l}5 \\
(15.6)\end{array}$ & $\begin{array}{l}16 \\
(50.0)\end{array}$ & $\begin{array}{l}11 \\
(34.4)\end{array}$ \\
\hline \multicolumn{2}{|l|}{ Total } & 1544 & 89 & 77 & 233 & 80 & 7 \\
\hline \multicolumn{2}{|c|}{ Percent $(\%)$} & 90.3 & 5.2 & 4.5 & 72.8 & 25.0 & 2.2 \\
\hline
\end{tabular}

Note: Figures in parentheses represent percentages $(\%)$

From Table 1, 62.5\% of the principals indicated that they performed the instructional role of giving demonstration lessons satisfactorily, $28.1 \%$ performed it fairly and $9.4 \%$ performed the role poorly. However, $67.8 \%$ of the teachers perceived that principals performed this role which corroborates the principals' claim. About $72 \%$ of the principals also performed the instructional role of summoning meetings of HODs satisfactorily, Twenty-five percent performed it fairly well while only one (3.1\%) never performed the role at all. $93 \%$ of the teachers however perceived that the principals perform this role. This shows that a larger percentage of teachers perceive that principals perform the role accordingly. About $63 \%$ of the 175 teachers agreed that principals are expected to perform the instructional role of organizing in-service training for teachers. However, only $15.6 \%$ of the principals were found to perform this role very well (satisfactorily), $50 \%$ performed it just a little, while $34.4 \%$ poorly performed the role.

In all, though $90.3 \%$ of the teachers expected that principals should perform all the listed instructional roles, $72.8 \%$ and $25 \%$ respectively of the principals satisfactorily and fairly performed the instructional roles. About $2.2 \%$ of the principals performed this role poorly. This implies that none of the principals poorly performed the three items of their instructional role suggest that the principals are performing the roles as perceived by the teachers.

Table2. Teachers perception of the principals' actual performance of their administrative roles

\begin{tabular}{|c|c|c|c|c|c|c|c|}
\hline \multirow[t]{3}{*}{$\mathbf{Q} / \mathbf{N}$} & \multirow[t]{3}{*}{ Administrative Role } & \multicolumn{3}{|c|}{ Teachers Perception } & \multicolumn{3}{|c|}{ Principals Actual Performance } \\
\hline & & Positive & Neutral & Negative & Satisfactory & Fair & Poor \\
\hline & & $\begin{array}{c}\text { Freq. } \\
(\%)\end{array}$ & $\begin{array}{l}\text { Freq. } \\
(\%)\end{array}$ & $\begin{array}{c}\text { Freq. } \\
(\%)\end{array}$ & $\begin{array}{c}\text { Freq. } \\
(\%)\end{array}$ & $\begin{array}{c}\text { Freq. } \\
(\%)\end{array}$ & $\begin{array}{c}\text { Freq. } \\
(\%)\end{array}$ \\
\hline 5 & Hold general staff meeting & $\begin{array}{l}167 \\
(97.6)\end{array}$ & $\begin{array}{l}2 \\
(1.2)\end{array}$ & $\begin{array}{l}2 \\
(1.2)\end{array}$ & $\begin{array}{l}29 \\
(90.6)\end{array}$ & $\begin{array}{l}3 \\
(9.4)\end{array}$ & - \\
\hline 8 & $\begin{array}{l}\text { Assign subject to teachers } \\
\text { according to qualification }\end{array}$ & $\begin{array}{ll}162 \\
(94.8)\end{array}$ & $\begin{array}{l}6 \\
(3.5)\end{array}$ & $\begin{array}{l}3 \\
(1.8)\end{array}$ & $\begin{array}{l}25 \\
(78.1)\end{array}$ & $\begin{array}{l}7 \\
(21.9)\end{array}$ & - \\
\hline 11 & $\begin{array}{l}\text { Periodically check attendance } \\
\text { registers }\end{array}$ & $\begin{array}{l}161 \\
(94.2)\end{array}$ & $\begin{array}{l}6 \\
(3.5)\end{array}$ & $\begin{array}{l}4 \\
(2.3)\end{array}$ & $\begin{array}{l}16 \\
(50.0)\end{array}$ & $\begin{array}{l}14 \\
(43.8)\end{array}$ & $\begin{array}{l}2 \\
(6.3)\end{array}$ \\
\hline
\end{tabular}


Appraisal of Principals' Role Performance and the Teachers' Perception of the Same Roles in Selected Public Schools in Osun State, Nigeria

\begin{tabular}{|c|c|c|c|c|c|c|c|}
\hline 14 & $\begin{array}{l}\text { Keep files of teaching and non- } \\
\text { teaching staff }\end{array}$ & \begin{tabular}{|l|}
156 \\
$(91.2)$
\end{tabular} & $\begin{array}{l}11 \\
(6.4)\end{array}$ & $\begin{array}{l}4 \\
(2.4)\end{array}$ & \begin{tabular}{|l|l}
28 \\
$(87.5)$
\end{tabular} & $\begin{array}{l}2 \\
(6.3)\end{array}$ & \begin{tabular}{|l|l}
2 \\
$(6.3)$
\end{tabular} \\
\hline 17 & $\begin{array}{l}\text { Show concern about discipline } \\
\text { of students }\end{array}$ & $\begin{array}{l}168 \\
(98.3)\end{array}$ & $\begin{array}{l}1 \\
(0.6)\end{array}$ & $\begin{array}{l}2 \\
(1.2) \\
\end{array}$ & $\begin{array}{l}31 \\
(96.9) \\
\end{array}$ & $\begin{array}{l}1 \\
(3.1) \\
\end{array}$ & - \\
\hline 20 & $\begin{array}{l}\text { Promote and encourage extra- } \\
\text { curricular activity }\end{array}$ & $\begin{array}{ll}157 \\
(91.8)\end{array}$ & \begin{tabular}{|l|}
5 \\
$(2.9)$ \\
\end{tabular} & $\begin{array}{l}9 \\
(5.3)\end{array}$ & \begin{tabular}{|l|l|}
18 \\
$(56.3)$
\end{tabular} & \begin{tabular}{|l|l|}
12 \\
$(37.5)$
\end{tabular} & $\begin{array}{l}2 \\
(6.3) \\
\end{array}$ \\
\hline 22 & \begin{tabular}{|llr}
$\begin{array}{l}\text { Make experienced graduate } \\
\text { teachers heads of departments } \\
\text { (HOD) }\end{array}$ & \\
\end{tabular} & $\begin{array}{l}158 \\
(92.4)\end{array}$ & $\begin{array}{l}6 \\
(3.5)\end{array}$ & $\begin{array}{l}7 \\
(4.1)\end{array}$ & $\begin{array}{l}28 \\
(87.5)\end{array}$ & $\begin{array}{l}4 \\
(12.5)\end{array}$ & - \\
\hline 23 & \begin{tabular}{|ll} 
Make HODs & supervise \\
instruction
\end{tabular} & \begin{tabular}{|l|l}
163 \\
$(95.4)$
\end{tabular} & $\begin{array}{l}5 \\
(2.9)\end{array}$ & $\begin{array}{l}3 \\
(1.8)\end{array}$ & $\begin{array}{l}27 \\
(84.4)\end{array}$ & $\begin{array}{ll}5 \\
(15.6)\end{array}$ & - \\
\hline 29 & $\begin{array}{ll}\text { Exercise authority when } \\
\text { occasion demands }\end{array}$ & \begin{tabular}{|l|}
152 \\
$(88.9)$ \\
\end{tabular} & $\begin{array}{l}15 \\
(8.8) \\
\end{array}$ & \begin{tabular}{|l|}
4 \\
$(2.3)$ \\
\end{tabular} & $\begin{array}{l}21 \\
(65.6) \\
\end{array}$ & $\begin{array}{ll}10 \\
(31.3) \\
\end{array}$ & \begin{tabular}{|l|l}
1 \\
$(3.1)$ \\
\end{tabular} \\
\hline 30 & $\begin{array}{l}\text { Encourage teachers to record only } \\
\text { what has been taught in the diary }\end{array}$ & \begin{tabular}{|l|l|}
163 \\
$(95.3)$
\end{tabular} & $\begin{array}{l}6 \\
(3.5)\end{array}$ & $\begin{array}{l}2 \\
(1.2)\end{array}$ & \begin{tabular}{|l|l|}
25 \\
$(78.1)$
\end{tabular} & $\begin{array}{l}7 \\
(21.9)\end{array}$ & - \\
\hline \multicolumn{2}{|c|}{ Total } & 1371 & 167 & 172 & 248 & 65 & 7 \\
\hline \multicolumn{2}{|c|}{ Percent $(\%)$} & 80.2 & 9.8 & 10.0 & 77.5 & 20.3 & 2.2 \\
\hline
\end{tabular}

Note: Figures in parentheses represent percentages (\%)

Principals' actual performance of their administrative role, as perceived by the teachers, showed that $97.6 \%$ of the teachers perceived that principals performed the role of holding general staff meeting in schools. However, $90.6 \%$ of the principals performed this role satisfactorily while $9.4 \%$ performed it fairly well. None of the principals performed the role poorly. $98.3 \%$ of the teachers perceived that principals show concern about discipline of students while majority $(96.9 \%)$ of the principals claimed that they performed this role satisfactorily. Only $3.1 \%$ of the principals performed the role fairly while none of them performed poorly. The cumulative percent revealed that though $80.2 \%$ of the teachers perceived that principals perform the listed administrative roles, $77.5 \%$ of the principals admitted performing these roles satisfactorily, $20.3 \%$ performed it fairly well, while $2.2 \%$ performed poorly. This suggests that majority of the principals in Osun state public secondary schools perform their administrative roles towards maintaining discipline of students in the schools.

Table3. Teachers perception of the principals' actual performance of their human relation roles

\begin{tabular}{|c|c|c|c|c|c|c|c|}
\hline \multirow[t]{3}{*}{$\mathbf{Q} / \mathbf{N}$} & \multirow[t]{3}{*}{ Human Relation Roles } & \multicolumn{3}{|c|}{ Teachers Perception } & \multicolumn{3}{|c|}{ Principals Actual Performance } \\
\hline & & Positive & Neutral & Negative & Satisfactory & Fair & Poor \\
\hline & & $\begin{array}{c}\text { Freq. } \\
(\%)\end{array}$ & $\begin{array}{l}\text { Freq. } \\
(\%)\end{array}$ & $\begin{array}{c}\text { Freq. } \\
(\%)\end{array}$ & $\begin{array}{l}\text { Freq. } \\
(\%)\end{array}$ & $\begin{array}{c}\text { Freq. } \\
(\%)\end{array}$ & $\begin{array}{c}\text { Freq. } \\
(\%)\end{array}$ \\
\hline 3 & $\begin{array}{l}\text { Show concern for teachers } \\
\text { welfare }\end{array}$ & $\begin{array}{l}163 \\
(95.3) \\
\end{array}$ & $\begin{array}{l}5 \\
(2.9)\end{array}$ & $\begin{array}{l}3 \\
(1.8)\end{array}$ & $\begin{array}{l}28 \\
(87.5)\end{array}$ & $\begin{array}{l}4 \\
(12.5) \\
\end{array}$ & - \\
\hline 6 & $\begin{array}{l}\text { Recognize and commend } \\
\text { satisfactory work }\end{array}$ & $\begin{array}{l}166 \\
(97.0)\end{array}$ & $\begin{array}{l}2 \\
(1.2) \\
\end{array}$ & $\begin{array}{l}3 \\
(1.8)\end{array}$ & $\begin{array}{l}30 \\
(93.8) \\
\end{array}$ & $\begin{array}{l}2 \\
(6.3)\end{array}$ & - \\
\hline 9 & $\begin{array}{l}\text { Encourage interdependence } \\
\text { among teachers }\end{array}$ & $\begin{array}{l}149 \\
(87.2)\end{array}$ & $\begin{array}{l}10 \\
(5.8)\end{array}$ & $\begin{array}{l}12 \\
(7.0)\end{array}$ & $\begin{array}{l}22 \\
(68.8)\end{array}$ & $\begin{array}{l}10 \\
(31.3)\end{array}$ & - \\
\hline 12 & $\begin{array}{l}\text { Keep teachers at ease and } \\
\text { happy }\end{array}$ & $\begin{array}{l}166 \\
(97.1)\end{array}$ & $\begin{array}{l}4 \\
(2.3)\end{array}$ & $\begin{array}{l}1 \\
(0.6)\end{array}$ & $\begin{array}{l}26 \\
(81.3)\end{array}$ & $\begin{array}{l}6 \\
(8.8)\end{array}$ & - \\
\hline 15 & $\begin{array}{l}\text { Participate in community } \\
\text { activities }\end{array}$ & $\begin{array}{l}105 \\
(61.4)\end{array}$ & $\begin{array}{l}36 \\
(21.1)\end{array}$ & $\begin{array}{l}30 \\
(17.5)\end{array}$ & $\begin{array}{l}10 \\
(31.3)\end{array}$ & $\begin{array}{l}18 \\
(56.3)\end{array}$ & $\begin{array}{l}4 \\
(12.5)\end{array}$ \\
\hline 18 & Promote staff harmony & $\begin{array}{l}167 \\
(97.3)\end{array}$ & $\begin{array}{l}2 \\
(1.2)\end{array}$ & $\begin{array}{l}2 \\
(1.2)\end{array}$ & $\begin{array}{l}28 \\
(87.5)\end{array}$ & $\begin{array}{l}4 \\
(12.5)\end{array}$ & - \\
\hline 21 & $\begin{array}{l}\text { Keep teachers secret from } \\
\text { students }\end{array}$ & $\begin{array}{l}156 \\
(91.3)\end{array}$ & $\begin{array}{l}2 \\
(1.2)\end{array}$ & $\begin{array}{l}13 \\
(7.6)\end{array}$ & $\begin{array}{l}29 \\
(90.6)\end{array}$ & $\begin{array}{l}3 \\
(9.4)\end{array}$ & - \\
\hline 24 & $\begin{array}{l}\text { Encourage teachers to build } \\
\text { positive professional attitude }\end{array}$ & $\begin{array}{l}166 \\
(97.1)\end{array}$ & $\begin{array}{l}5 \\
(2.9)\end{array}$ & - & $\begin{array}{l}21 \\
(65.6)\end{array}$ & $\begin{array}{l}11 \\
(34.4)\end{array}$ & - \\
\hline 26 & $\begin{array}{l}\text { Seek the advice of the } \\
\text { HODs before budgeting }\end{array}$ & $\begin{array}{l}153 \\
(89.5)\end{array}$ & $\begin{array}{l}10 \\
(5.8)\end{array}$ & $\begin{array}{l}8 \\
(4.7)\end{array}$ & $\begin{array}{l}19 \\
(59.4)\end{array}$ & $\begin{array}{l}12 \\
(37.5)\end{array}$ & $\begin{array}{l}1 \\
(3.1)\end{array}$ \\
\hline 27 & $\begin{array}{l}\text { Settle dispute among } \\
\text { teachers }\end{array}$ & $\begin{array}{l}153 \\
(89.5)\end{array}$ & $\begin{array}{l}13 \\
(7.6)\end{array}$ & $\begin{array}{l}5 \\
(3.0) \\
\end{array}$ & $\begin{array}{l}20 \\
(62.5)\end{array}$ & $\begin{array}{l}10 \\
(31.3)\end{array}$ & $\begin{array}{l}2 \\
(6.3) \\
\end{array}$ \\
\hline \multicolumn{2}{|c|}{ Total } & 1544 & 89 & 77 & 233 & 80 & 7 \\
\hline \multicolumn{2}{|c|}{ Percent (\%) } & 90.2 & 5.2 & 4.5 & 72.8 & 25.0 & 2.2 \\
\hline
\end{tabular}

Note: Figures in parentheses represent percentages (\%) 
While $95.3 \%$ of the 175 teachers perceived that principals performed the human relation role of showing concern for teachers' welfare, $87.5 \%$ of the principals agreed to performing this role, $12.5 \%$ performed the role fairly well while none of the principals recorded poor performance of this role. Almost all $97 \%$ of the teachers perceived that principals performed the role of recognizing and commending teachers for satisfactory work. However, $93.8 \%$ of the principals claimed that they performed this role satisfactorily while $6.3 \%$ performed it fairly and none poorly. Results of analysis of the human relation role of participating in community activities showed that while $61.4 \%$ of the teachers agreed that principals performed this role, $31.3 \%$ indicated satisfactory performance while $56.3 \%$ performed it fairly. Four $(12.5 \%)$ of the principals recorded poor performance. In general, $90.2 \%$ of the teachers perceived that the principals performed their human relation roles, $72.8 \%$ of the principals claimed performing this role satisfactorily. $25 \%$ performed it fairly while only $2.2 \%$ performed poorly.

Table4. Teachers' rating of the principals' actual performance of their instructional roles

\begin{tabular}{|c|c|c|c|c|}
\hline \multirow[t]{3}{*}{$\mathbf{Q} / \mathbf{N}$} & \multirow[t]{3}{*}{ Instructional Role } & \multicolumn{3}{|c|}{ Teachers Actual Rating of Principals' Performance } \\
\hline & & Positive & Neutral & Negative \\
\hline & & $\begin{array}{c}\text { Freq. } \\
(\%)\end{array}$ & $\begin{array}{c}\text { Freq. } \\
(\%)\end{array}$ & $\begin{array}{c}\text { Freq. } \\
(\%)\end{array}$ \\
\hline 1 & Give demonstration lessons & $\begin{array}{l}58 \\
(33.9)\end{array}$ & $\begin{array}{l}69 \\
(40.4)\end{array}$ & $\begin{array}{l}44 \\
(25.7)\end{array}$ \\
\hline 2 & $\begin{array}{lll}\text { Provide } & \text { badly } & \text { needed } \\
\text { instructional materials } & \end{array}$ & $\begin{array}{l}81 \\
(47.4)\end{array}$ & $\begin{array}{l}75 \\
(43.9)\end{array}$ & $\begin{array}{l}15 \\
(8.8)\end{array}$ \\
\hline 4 & $\begin{array}{l}\text { Ensure that teachers improve on } \\
\text { the use of instructional materials }\end{array}$ & $\begin{array}{l}77 \\
(45.0)\end{array}$ & $\begin{array}{l}87 \\
(50.9)\end{array}$ & $\begin{array}{l}7 \\
(4.1)\end{array}$ \\
\hline 7 & $\begin{array}{l}\text { Organize orientation course for } \\
\text { new teachers }\end{array}$ & $\begin{array}{l}40 \\
(23.4)\end{array}$ & $\begin{array}{l}65 \\
(38.0)\end{array}$ & $\begin{array}{l}66 \\
(38.6)\end{array}$ \\
\hline 10 & $\begin{array}{l}\text { Encourage teachers to attend } \\
\text { seminars }\end{array}$ & $\begin{array}{l}77 \\
(45.0)\end{array}$ & $\begin{array}{l}73 \\
(42.7)\end{array}$ & $\begin{array}{l}21 \\
(12.3)\end{array}$ \\
\hline 13 & $\begin{array}{l}\text { Undertake } \\
\text { visitation }\end{array}$ & $\begin{array}{l}45 \\
(26.3)\end{array}$ & $\begin{array}{l}80 \\
(46.8)\end{array}$ & $\begin{array}{l}46 \\
(26.9)\end{array}$ \\
\hline 16 & Help the inexperienced teachers & $\begin{array}{l}51 \\
(29.8)\end{array}$ & $\begin{array}{l}86 \\
((50.3)\end{array}$ & $\begin{array}{l}34 \\
(19.9)\end{array}$ \\
\hline 19 & $\begin{array}{l}\text { Occasionally collect random } \\
\text { samples of students' exercise books }\end{array}$ & $\begin{array}{l}68 \\
(39.8) \\
\end{array}$ & $\begin{array}{l}76 \\
(44.4) \\
\end{array}$ & $\begin{array}{l}27 \\
(15.8)\end{array}$ \\
\hline 25 & $\begin{array}{l}\text { Summon meetings of } \\
\text { departmental heads to coordinate } \\
\text { instructions }\end{array}$ & $\begin{array}{l}110 \\
(64.3)\end{array}$ & $\begin{array}{l}55 \\
(32.2)\end{array}$ & $\begin{array}{l}6 \\
(3.5)\end{array}$ \\
\hline 28 & $\begin{array}{l}\text { Organize in-service training for } \\
\text { teachers }\end{array}$ & $\begin{array}{l}35 \\
(20.5)\end{array}$ & $\begin{array}{l}64 \\
(37.4)\end{array}$ & $\begin{array}{l}72 \\
(42.1)\end{array}$ \\
\hline \multicolumn{2}{|c|}{ Total } & 642 & 730 & 338 \\
\hline \multicolumn{2}{|c|}{ Percent $(\%)$} & 37.5 & 42.7 & 19.8 \\
\hline
\end{tabular}

Note: Figures in parentheses represent percentages (\%)

The results of teachers' actual rating of principals' performance of their instructional, administrative and human relation roles are presented in Table 4. From the Table, about $34 \%$ of the teachers rated the principals to be performing the role of giving demonstration lessons, $40.4 \%$ were undecided while $25.7 \%$ recorded negative responses to show that principals did not perform this role. Also, $23.4 \%$ of the teachers rated the principals high performing the role of organizing orientation course for new teachers, $38 \%$ were not certain if the principals actually performed this role while $38.6 \%$ of the teachers responded that principals did not perform this role. On the instructional role of summoning meetings of departmental heads to coordinate instructions, $64.3 \%$ of the teachers agreed that the principals actually performed this role. However, about $3.5 \%$ had a negative view while $32.2 \%$ were undecided as to whether the principals performed this role or not. The instructional role of organizing in-service training for teachers was rated positively by $20.5 \%$ of the teachers as being performed by the principals while $42.1 \%$ had negative responses. About $37.4 \%$ however claimed not to be sure of principals' performance of this role. The overall percentage of teachers' actual rating of principals' performance of instructional role showed that $37.5 \%$ of the teachers agreed that principals actually performed this role, $42.7 \%$ were undecided while $19.8 \%$ had a negative view. 
Appraisal of Principals' Role Performance and the Teachers' Perception of the Same Roles in Selected Public Schools in Osun State, Nigeria

Table5. Teachers' rating of the principals' actual performance of their administrative roles

\begin{tabular}{|c|c|c|c|c|}
\hline \multirow[t]{3}{*}{$\mathbf{Q} / \mathbf{N}$} & \multirow[t]{3}{*}{ Administrative Role } & \multicolumn{3}{|c|}{ Teachers Actual Rating of Principals' Performance } \\
\hline & & Positive & Neutral & Negative \\
\hline & & Freq. (\%) & Freq. (\%) & Freq. (\%) \\
\hline 5 & Hold general staff meeting & $\begin{array}{l}132 \\
(77.2)\end{array}$ & $\begin{array}{l}35 \\
(20.5)\end{array}$ & $\begin{array}{l}4 \\
(2.3)\end{array}$ \\
\hline 8 & $\begin{array}{l}\text { Assign subject to teachers } \\
\text { according to qualification }\end{array}$ & $\begin{array}{l}126 \\
(73.7)\end{array}$ & $\begin{array}{l}43 \\
(25.1)\end{array}$ & $\begin{array}{l}2 \\
(1.2)\end{array}$ \\
\hline 11 & $\begin{array}{l}\text { Periodically check attendance } \\
\text { registers }\end{array}$ & $\begin{array}{l}104 \\
(60.8)\end{array}$ & $\begin{array}{l}57 \\
(33.3)\end{array}$ & $\begin{array}{l}10 \\
(5.8)\end{array}$ \\
\hline 14 & $\begin{array}{l}\text { Keep files of teaching and non- } \\
\text { teaching staff }\end{array}$ & $\begin{array}{l}128 \\
(74.9)\end{array}$ & $\begin{array}{l}40 \\
(23.4)\end{array}$ & $\begin{array}{l}3 \\
(1.8)\end{array}$ \\
\hline 17 & $\begin{array}{l}\text { Show concern about discipline } \\
\text { of students }\end{array}$ & $\begin{array}{l}151 \\
(88.3)\end{array}$ & $\begin{array}{l}19 \\
(11.1)\end{array}$ & $\begin{array}{l}1 \\
(0.6)\end{array}$ \\
\hline 20 & $\begin{array}{l}\text { Promote and encourage extra- } \\
\text { curricular activity }\end{array}$ & $\begin{array}{l}94 \\
(55.0)\end{array}$ & $\begin{array}{l}62 \\
(36.3)\end{array}$ & $\begin{array}{l}15 \\
(8.8)\end{array}$ \\
\hline 22 & $\begin{array}{l}\text { Make experienced graduate } \\
\text { teachers heads of departments } \\
\text { (HOD) }\end{array}$ & $\begin{array}{l}136 \\
(79.5)\end{array}$ & $\begin{array}{l}35 \\
(20.5)\end{array}$ & - \\
\hline 23 & $\begin{array}{l}\text { Make HODs } \\
\text { instruction }\end{array}$ & $\begin{array}{l}127 \\
(74.3)\end{array}$ & $\begin{array}{l}42 \\
(24.6)\end{array}$ & $\begin{array}{l}2 \\
(1.2)\end{array}$ \\
\hline 29 & $\begin{array}{l}\text { Exercise authority when } \\
\text { occasion demands }\end{array}$ & $\begin{array}{l}108 \\
(63.2)\end{array}$ & $\begin{array}{l}62 \\
(36.3)\end{array}$ & $\begin{array}{l}1 \\
(0.6)\end{array}$ \\
\hline 30 & $\begin{array}{l}\text { Encourage teachers to record } \\
\text { only what has been taught in the } \\
\text { diary }\end{array}$ & $\begin{array}{l}107 \\
(62.6)\end{array}$ & $\begin{array}{l}61 \\
(35.7)\end{array}$ & $\begin{array}{l}3 \\
(1.8)\end{array}$ \\
\hline \multicolumn{2}{|c|}{ Total } & 1213 & 456 & 41 \\
\hline \multicolumn{2}{|c|}{ Percent (\%) } & 70.9 & 26.7 & 2.4 \\
\hline
\end{tabular}

Note: Figures in parentheses represent percentages (\%)

The results of analysis on principals' administrative role showed that about $77.2 \%$ of the teachers supported that their principals performed the role of holding general staff meeting. About $20.5 \%$ of them claimed to be uncertain of whether the principals performed this role or not, while $2.3 \%$ of them had a negative response that their principals never performed the role at all. Also $74.9 \%$ of the teachers positively responded that their principals performed the administrative role of keeping files of teaching and non-teaching staff, $23.4 \%$ were not certain, while $1.8 \%$ claimed the principals never performed this role. About $88.3 \%$ of the teachers indicated that principals actually performed the role of showing concern for students' discipline, $11.1 \%$ were undecided, while only one $(0.6 \%)$ signified poor performance of this role by the principals. The overall percentage rating of the principals' performance of their administrative role showed that $70.9 \%$ of the teachers support the performance of this role by the principals, $26.7 \%$ were not certain, while $2.4 \%$ claimed poor performance.

Table6. Teachers' rating of the principals' actual performance of their human relation roles

\begin{tabular}{|c|c|c|c|c|}
\hline \multirow[t]{3}{*}{ QN } & \multirow[t]{3}{*}{ Human Relation Roles } & \multicolumn{3}{|c|}{ Teachers Actual Rating of Principals' Performance } \\
\hline & & Positive & Neutral & Negative \\
\hline & & Freq. $(\%)$ & Freq. $(\%)$ & Freq. $(\%)$ \\
\hline 3 & $\begin{array}{l}\text { Show concern for teachers } \\
\text { welfare }\end{array}$ & $\begin{array}{l}134 \\
(78.4)\end{array}$ & $\begin{array}{l}36 \\
(21.1)\end{array}$ & $\begin{array}{l}1 \\
(0.6)\end{array}$ \\
\hline 6 & $\begin{array}{l}\text { Recognize and commend } \\
\text { satisfactory work }\end{array}$ & $\begin{array}{l}121 \\
(70.8) \\
\end{array}$ & $\begin{array}{l}45 \\
(26.3)\end{array}$ & $\begin{array}{l}5 \\
(2.9)\end{array}$ \\
\hline 9 & $\begin{array}{l}\text { Encourage interdependence } \\
\text { among teachers }\end{array}$ & $\begin{array}{l}100 \\
(58.5)\end{array}$ & $\begin{array}{l}67 \\
(39.2)\end{array}$ & $\begin{array}{l}4 \\
(2.3)\end{array}$ \\
\hline 12 & Keep teachers at ease and happy & $\begin{array}{l}128 \\
(74.9)\end{array}$ & $\begin{array}{l}39 \\
(22.8)\end{array}$ & $\begin{array}{l}4 \\
(2.3)\end{array}$ \\
\hline 15 & $\begin{array}{l}\text { Participate in community } \\
\text { activities }\end{array}$ & $\begin{array}{l}36 \\
(21.1)\end{array}$ & $\begin{array}{l}98 \\
(57.3)\end{array}$ & $\begin{array}{l}37 \\
(21.6)\end{array}$ \\
\hline 18 & Promote staff harmony & $\begin{array}{l}143 \\
(83.6)\end{array}$ & $\begin{array}{l}27 \\
(15.8)\end{array}$ & $\begin{array}{l}1 \\
(0.6)\end{array}$ \\
\hline
\end{tabular}


Appraisal of Principals' Role Performance and the Teachers' Perception of the Same Roles in Selected Public Schools in Osun State, Nigeria

\begin{tabular}{|l|l|l|l|l|}
\hline 21 & $\begin{array}{l}\text { Keep teachers secret from } \\
\text { students }\end{array}$ & $\begin{array}{l}127 \\
(74.3)\end{array}$ & $\begin{array}{l}36 \\
(21.1)\end{array}$ & $\begin{array}{l}8 \\
(4.7)\end{array}$ \\
\hline 24 & $\begin{array}{l}\text { Encourage teachers to build } \\
\text { positive professional attitude }\end{array}$ & $\begin{array}{l}107 \\
(62.2)\end{array}$ & 60 & 4 \\
& $\begin{array}{l}(35.1) \\
(4.7)\end{array}$ \\
\hline 26 & $\begin{array}{l}\text { Seek the advice of the HODs } \\
\text { before budgeting }\end{array}$ & 104 & 59 & 8 \\
$(60.8)$ & $(34.5)$ & $(4.7)$ \\
\hline 27 & Settle dispute among teachers & 103 & 52 & 16 \\
& $(60.2)$ & $(30.4)$ & $(9.4)$ \\
\hline Total & 1103 & 519 & 88 \\
\hline Percent (\%) & 64.5 & 30.4 & 5.1 \\
\hline
\end{tabular}

Note: Figures in parentheses represent percentages (\%)

Teachers rating of principals' human relation role (Table 6) showed that $78.4 \%$ of the principals performed the role of showing concern for teachers welfare, $21 \%$ recorded uncertainty in principals performance of this role, while only $0.6 \%$ had a negative response. Teachers rating of principals' role of recognizing and commending satisfactory work showed that $70.8 \%$ of the teachers agreed that principals performed this role, $26.3 \%$ were undecided while $2.9 \%$ of the teachers claimed nonperformance by the principals. Many of the teachers 37 (21.6\%) claimed that their principals never performed the role of participating in community activities, $98(57.3 \%)$ teachers agreed that principals have performed the role, while $36(21.1 \%)$ were undecided on whether the principals performed this role or not. In the area of settling dispute among teachers, $60.2 \%$ of the teachers rated the principals positively as performing this role, $30.4 \%$ were undecided on whether principals should be rated positively or negatively, while $9.6 \%$ of the teachers revealed that principals never performed this role. The teachers rating of the entire human relation role showed about $65 \%$ of the teachers agreed that principals performed this role, $30.4 \%$ of the teachers were undecided, and $5.1 \%$ did not agree that principals performed this role. The high percentage of teachers who were undecided in their views on principals performance of their instructional $(42.7 \%)$, administrative $(26.7 \%)$ and human relation roles $(30.4 \%)$ showed that most teachers are not really been observant in noting/monitoring the principals activities in the schools.

\section{DisCUSSION OF FINDINGS}

Although a large proportion of teachers perceived that the principals performed their instructional, administrative, and human relation roles, variations exist in principals' actual performance of these roles. For example, while $90.3 \%$ of the teachers perceived that principals performed the instructional role, only $72.8 \%$ actually performed this role. Also, $80.2 \%$ and $90.2 \%$ of the teachers respectively expected the principals to perform the administrative and human relation roles but only $77.5 \%$ and $72.8 \%$ of the principals actually performed these roles. In general, less than $40 \%$ of the principals' role set i.e. the teachers agreed that the principals actually performed their instructional role, while $42.7 \%$ teachers were undecided on the performance of this role by the principals. However, a slightly higher percentage of teachers (70.9\%) indicated that the principals performed their administrative role satisfactorily while $64.5 \%$ claimed the same for the human relation role. The percentage of teachers that were undecided on the principals' performance of these roles was $26.7 \%$ and $30.4 \%$ respectively for the administrative and human relation roles.

\section{CONCLUSION}

Based on the results obtained, the following conclusions were reached:

- The variation in teachers' perception and actual performance of principals' roles shows that there is still room for principals to improve upon the actual performance of their expected instructional, administrative and human relation roles in Osun state public secondary schools. This implies that there is need for formal orientation for new principals to ensure adequate knowledge of their role performance in secondary school.

- The cumulative percent of principals who actually performed the instructional, administrative and human relation roles in the schools was less than the percentage expectation of their performance by their role set (teachers). The general view of the principals' role set (teachers) on the principals' actual performance of his roles is that principals perform more of administrative role, followed by human relation role while the instructional role comes last. 
This is not in consonance with the views of Adaralegbe (1971) and Bamberger and Hasgall (1995) who pointed out that instructional leadership is the most important of the multiple roles of the principal.

- The high percentage of the principals' roles set (teachers) who were undecided on principals performance of their instructional administrative and human relation roles, showed that most teachers in the schools exhibit a non-chalant behaviour in noting/monitoring the activities of their principals in the schools. This implies that the principal's administrative style and human relation role is affecting the attitude of the teachers negatively in school by their nonchalant behaviour.

\section{IMPLICATIONS}

The findings from this study have implications for stakeholders in the Education Industry especially the principals, teachers, policy-makers as well as government. It is important to note that effective performance of principals' role is capable of enhancing the academic performance of the students in secondary schools. To be able to achieve the desired goals, principals must be provided with relevant resources (such as grant-in-aid, instructional materials, laboratory equipment, teaching aids. etc.) and government should ensure that principals improve on their role performance. This implies that there is a need for adequate in-service training of new principals and re-training of those already on the job to ensure adequate knowledge of their expected roles. This corroborates the view of Cooke and Dunhill (1966) who stated that as a leader, a principal must plan, coordinate and supervise the affairs of the school so that it runs smoothly. To be able to do this, they claimed that, he, must keep himself up- todate professionally and unless he reads relevant books and he is aware of new ideas and techniques, as well as the old tested ones, he runs the risk of becoming too rigid and fixed in his ways.

\section{REFERENCES}

[1] Adaralegbe, A. (1983). "Secondary Education in Nigeria". Nigerian Education: Trends and Issues. (ed.). University of Ife Press Ltd., Ife.

[2] Adelabu, M.A. (1981). "Role conflict of selected secondary school principals in Oyo state". An unpublished M.A. thesis, Department of Educational Administration and Planning, O.A.U., Ile-Ife, Nigeria.

[3] Adeyegbe, S.O. (1992): "The West African Examinations Council (WAEC) and curriculum development". In Ivowi, U.M.O. (ed.). Curriculum Development in Nigeria, Ibadan: Sam Bookman.

[4] Adeyemi T.O (2006) Fundamentals of Educational Management Lagos: Atlantic Associated Publishers pp 21-60.

[5] Afolabi, F.O. (1998). "An appraisal of student welfare administration in selected secondary schools in Ondo state, Nigeria." Ife Journal of Educational Studies, O.A.U. Ile-Ife: 1(1) pp. 153-154.

[6] Ajeyalemi, D. and A. Ejiogu (1987). "Issues, problems and prospects in Nigerian Education". In Emergent Issues in Nigerian Education. (ed.) Vol.1: 1-10.

[7] Akerele S.A. 2007; Principals leadership styles and Teachers' performance in Lagos State public secondary schools "Unpublished M.Ed Thesis, University of Ado-Ekiti, Nigeria pp 110-124.

[8] Bacharach, S.B., Bamberger, P. and Conley, S.C. (1996). "Negotiating the 'see-saw' of managerial strategy: a resurrection of the study of professionals in organizational theory". Research in the Sociology of organizations. Pp. 217-23

[9] Biddle, J.W. and Thomas, B.J. (1966). Role concepts and research. New York: John Wiley and So

[10] Cooke, D. and Dunhill, J. (1966). School administration and management. London: University of London Press.

[11] Deighton, L.A. (1971). Encyclopedia of education. (ed.) Vol.7. New York: Macmillan Company and the Free Press.

[12] Fonaldson, G.A. (2007). Learning to lead: The dynamics of the high school principalship. Westport, CT: Greenwood

[13] Moye G. P (2014). Effects of school variables on the Assurance of Quality Learning Outcomes in schools, International Journal of Learning Teaching and Educational Research; vol. 9 No 1 December 2014.

[14] Niewman, L. and Hughes, J.W. (1951). "The problem of the concept of role: A resurvey of literature", Social Forces, Vol.33, No.2.

[15] Oluwatoyin F.I. (2003) Managing Behaviour and Performance in organization. Lagos: Sp. Sege Prints, Nigeria, pp 34-45. 
[16] Nworgu, B.G. (1991). Educational research: Basic issues and methodology. Wisdom publishers Ltd., Ibadan, Nigeria.

[17] Ogbiji S. A (2014). A boom in Private Sector Education in Cross River state, Nigeria; Causes and Consequences on the Educational System; Journal of Educational and Social Research, Vol. 4 No 6 RomeItaly, September 2014.

[18] Oyewole, B.K. and Alonge,H.O.(2013) Principals Instructional Supervisory Role Performance and Teachers' Motivation in Ekiti Central Senatorial District of Ekiti State ,Nigeria; Journal of Education and Social Research; Vol.3(2) May 2013.

[19] Rizzo, J., House, R. and Lirtzman, S. (1970). "Role conflict and ambiguity in complex organizations". Administrative Science Quarterly. Vol. 15, No.2. pp 150-63.

[20] Shuaibu, M. (2016). Supervisory Role of Principal on Teacher Duties in Secondary Schools. 200 pages of Finest Quality paper + 90 GSM

[21] Stroke, J.H. (2006). A position in transition National Association of School Principals 67 (5); 32-33.

[22] Uwazurike, C.N. (1992). Theories of educational leadership: implication for the Nigerian Educational Administrator in training and utilization of Educational Planners and administrators in Africa, Edited by Nwagwu, N.A. et al. The Nigerianjh Association for Educational Administrators and Planners (NAEAP) publication.

[23] Walker, A. (2015) Review of research on School Principal leadership in Mainland China, 1998-2013; Continuity and Change. 53 Issue: 4 https://doi.org/10.1108/FEA05-2014

\section{AUTHOR'S BIOGRAPHY}

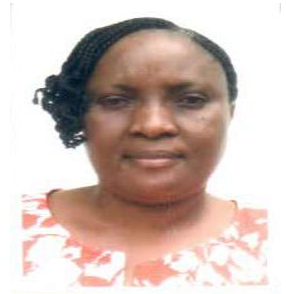

Felicia Bosede Bamire, has Ph.D.(Educational Administration) from Obafemi Awolowo University, Ile-Ife, Osun State, Nigeria. She currently works with the Teaching Service Commission of Osun state, a member of the English Association of Nigeria and the Teachers' Registration Council of Nigeria, Ile-Ife and has attended different conferences, seminars and workshops and published in both local and international journals in Educational Administration.

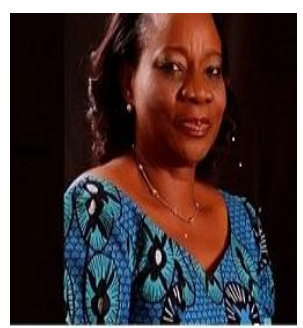

Modupe Adelabu is a Professor of Educational Administration and Planning, Obafemi Awolowo University, Ile-Ife, Nigeria. Herspecializeson policy and personnel issues in Education. She has served as external examiner for many Universities, attended several conferences, workshops and seminars and has over 30 publications. She had served as Council member in institutions such as National Teachers' Institute and Joseph Ayo Babalola University, Nigeria.

Citation: Dr. F. B. Bamire, Prof. M. A. Adelabu . "Appraisal of Principals' Role Performance and the Teachers' Perception of the Same Roles in Selected Public Schools in Osun State, Nigeria". International Journal of Humanities Social Sciences and Education (IJHSSE), vol 5, no. 6, 2018, pp. 48-57. doi: http://dx.doi.org/10.20431/2349-0381.0506007.

Copyright: (C) 2018 Authors. This is an open-access article distributed under the terms of the Creative Commons Attribution License, which permits unrestricted use, distribution, and reproduction in any medium, provided the original author and source are credited. 